\title{
Challenges for the care delivery for critically ill COVID-19 patients in developing countries: the Brazilian perspective
}

\author{
Jorge I. F. Salluh ${ }^{1,2^{*}}$, Thiago Lisboa ${ }^{3,4}$ and Fernando A. Bozza ${ }^{1,5}$
}

\section{Background}

The delivery of critical care is a major challenge for developing countries [1]. The inequity of access to an ICU bed, heterogeneous triage policies, a low staff/patient ratio and suboptimal adherence to evidence-based practices contribute to disproportionally high mortality of sepsis and acute respiratory distress syndrome in these countries [2-5]. In addition, limited step-down and specialized ward beds' availability further widens the gap between critical and non-critical care inside hospitals.

As the COVID-19 pandemic spreads through the world, developing countries are challenged with the surge of pneumonia cases where up to $30 \%$ of all hospitalized cases will require ICU admission [6]. In August 2020, Brazil is a hotspot of COVID-19 with more than 100,000 deaths. Other Latin American countries such as Mexico, Peru, Colombia, and Chile are also among the 10 countries with most cases worldwide. Several factors seem to have contributed to the dramatic progress of the epidemic in the country. Initial measures of social distancing were adopted at the beginning of the epidemic in several states. However, the lack of central coordination and, at a certain point, the denial of the pandemic by a populist government meant that more effective measures such as lockdown were not adopted whereas use of unproven therapies such as hydroxychloroquine was encouraged. Also, the low availability of tests and progression towards the interior and

\footnotetext{
* Correspondence: jorgesalluh@gmail.com

${ }^{1}$ Department of Critical Care and Postgraduate Program in Translational Medicine, D'Or Institute for Research and Education (IDOR), Rua Diniz Cordeiro, 30 - 30 andar, Rio de Janeiro 22281-100, Brazil

${ }^{2}$ Programa de Pós-Graduação em Clínica Médica, Universidade Federal do Rio de Janeiro, Rio de Janeiro, Brazil

Full list of author information is available at the end of the article
}

peripheries of large cities made the epidemic hard to control causing overwhelming hospitals and ICUs.

\section{What are the challenges?}

Despite its high absolute number of ICU beds [7], even in comparison with western European countries, the heterogeneous regional distribution and payor-based access are major barriers for a more equitable delivery of critical care. Although increases in the number of ICU beds were recently made across the country as preparation for the pandemic, they are still insufficient to compensate regional differences (the North region has 50\% fewer ICU beds per capita as compared with the Southeast) or the imbalances between the public and private sector. The number of public ICU beds per capita is $72 \%$ lower than private sector ones, and only $22 \%$ of the population has access to private healthcare.

Additionally, ICU staffing is an important shortcoming in the COVID-19 pandemic in developing countries. First, the ICU staffing can be considered low compared to developed countries, the current national norm defines a minimum of 1 nurse for each 10 ICU beds, and nursing technicians are the central workforce in several ICUs. Add the fact of the significant turnover due to a large number of healthcare professionals who are sick due to COVID-19, and the need for healthcare personnel with ICU training increases with the new ICU beds implemented for the pandemic. Other aspect to take in consideration is also the increasing complexity due to changes in case-mix of ICUs, where a large number of patients with multi-organ failure surge simultaneously. Data from the Brazilian ICU registry (www. utisbrasileiras.com/en that covers $1 / 3$ of all ICU beds in the country) shows in 2019 an average of mechanical ventilation rates around $19 \%$ and an increase to $41 \%$ 
with COVID-19 [8]. This sudden shift in case-mix and increases in severity of illness of ICU patients could partially explain the high mortality rates for mechanically ventilated patients with COVID-19. The recently published results of the registry, in 13,941 COVID-19 patients requiring ICU admission, show a mortality rate of $32 \%$ for all patients and $67 \%$ for those requiring MV. This high mortality represents an excess even if compared to the more recent data on sepsis epidemiology, where rates were approximately 55\% [4]. Recently, clinical characteristics and outcomes of COVID-19 patients from National Registries of critical care in LMICs (Brazil, Argentina, Sri Lanka, India) and HICs (Australia, New Zealand, Netherlands) were made publicly available by an international benchmarking initiative (icubenchmarking.com). Overall ICU mortality rates are comparable (ranging from 26 to 33\%, except for Australia/new Zealand with rates of $7.8 \%$ ). However, when the mortality of ventilated patients is analyzed, it tends to be higher in LMICs.
In Brazil, lessons learned from the pre-COVID-19 period may be helpful and represent actionable information that hopefully can be translated into improved outcomes. Several studies performed in Brazilian ICUs demonstrate that there are opportunities to improve the quality of care. If the low baseline adherence to protective ventilation in ARDS [9, 10], sepsis protocols (less than $60 \%$ of patients) [5], or light sedation (less than $40 \%$ of patients) [9] may be seen as bad news, they also represent potentially modifiable factors (Fig. 1). Recent studies demonstrate that the use of quality improvement (QI) interventions is associated with improved outcomes in LMICs. Brazilian data shows that interventions such as early sepsis triage and treatment [5], the use of protocols for the prevention of ICU-acquired complications, and organizational changes (i.e., promoting autonomy for ICU nurses or adding a pharmacist to the staff) [11, 12] are associated with lower hospital mortality and lengths of stay. In addition, the use of a structured checklist during multidisciplinary rounds and the

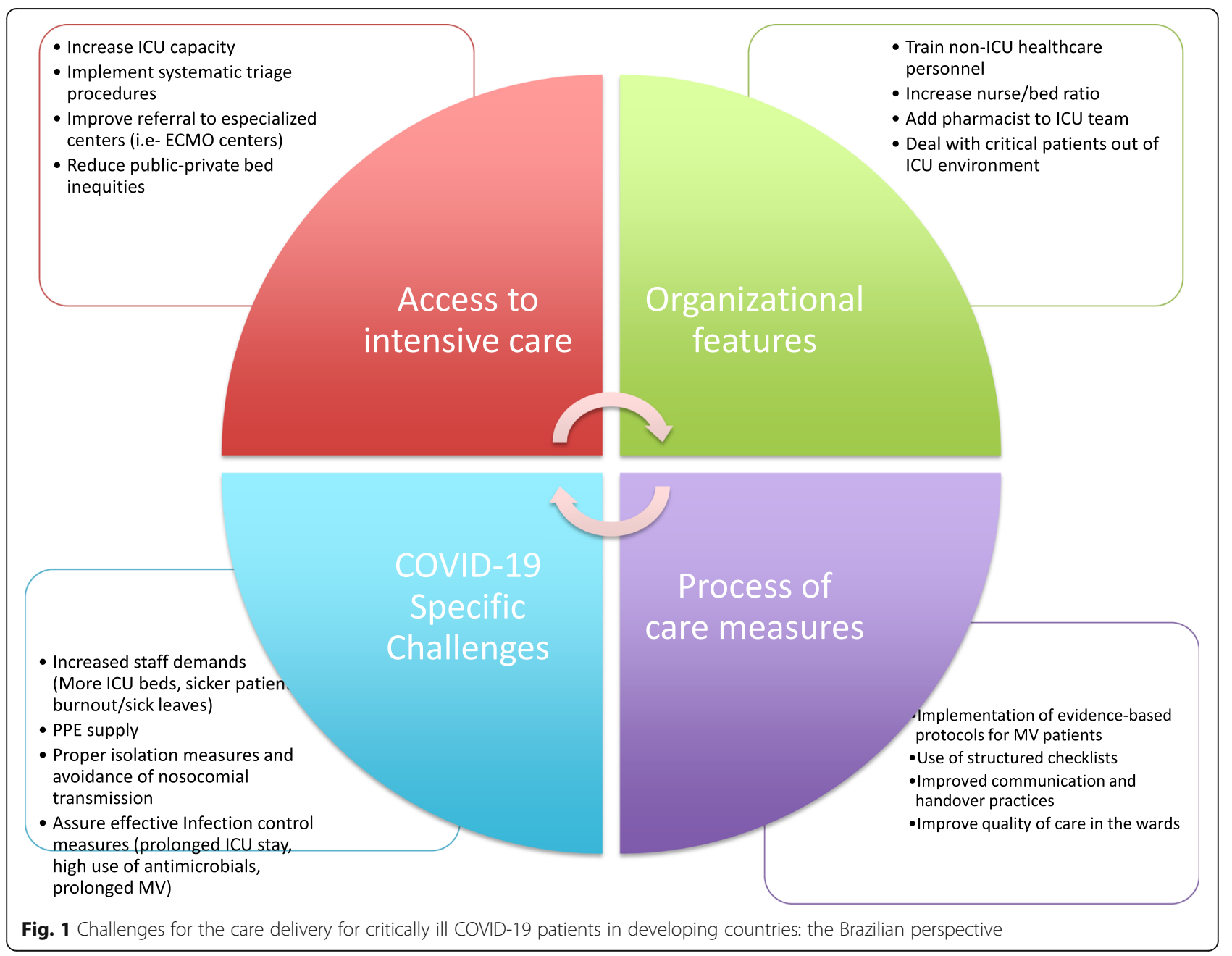


presence of an intensivist may improve adherence to sedation and protective ventilation protocols, both key factors to decrease the duration of ICU stay and to improve survival $[13,14]$.

\section{Conclusion}

In conclusion, there are no easy solutions and developing countries such as Brazil need to fix the system from "access to bedside care" in order to improve outcomes during and after the pandemic. In this regard, better triage procedures and improved ward care could help to get the right patients to the ICU on time. Also, providing better ward care could minimize long-term complications due to physical and cognitive impairment in this population. Using proven quality improvement interventions should be \#1 priority in the ICU as they represent a cost-effective strategy that usually does not require fancy technology or expensive drugs.

\section{Acknowledgements}

None

\section{Authors' contributions}

JIFS, TL, and FAB contributed to the conception, writing, and approval of the final manuscript.

\section{Funding}

Dr. Salluh is supported in part by individual research grants from CNPq and FAPERJ.

\section{Availability of data and materials}

N/A

\section{Ethics approval and consent to participate}

N/A

\section{Consent for publication}

The authors revise and approved the final version of manuscript.

\section{Competing interests}

None to declare

\section{Author details}

'Department of Critical Care and Postgraduate Program in Translational Medicine, D'Or Institute for Research and Education (IDOR), Rua Diniz Cordeiro, 30 - $3^{\circ}$ andar, Rio de Janeiro 22281-100, Brazil. ${ }^{2}$ Programa de Pós-Graduação em Clínica Médica, Universidade Federal do Rio de Janeiro, Rio de Janeiro, Brazil. ${ }^{3}$ Critical Care Department and Programa de Pós-Graduação em Ciencias Pneumologicas, Hospital de Clinicas de Porto Alegre, Universidade Federal do Rio Grande do Sul, Porto Alegre, Brazil. ${ }^{4}$ Instituto de Pesquisa Hospital do Coração - HCor, São Paulo, Brazil. ${ }^{5}$ Critical Care Lab, National Institute of Infectious Disease Evandro Chagas, Oswaldo Cruz Foundation, Rio de Janeiro, Brazil.

Received: 19 July 2020 Accepted: 4 September 2020

Published online: 30 September 2020

\section{References}

1. Schultz MJ, Dunser MW, Dondorp AM, Adhikari NK, lyer S, Kwizera A et al Global Intensive Care Working Group of the European Society of Intensive Care Medicine. Current challenges in the management of sepsis in ICUs in resource-poor settings and suggestions for the future. Intensive Care Med 2017;:43(5):612-624.

2. Salluh JIF, Soares M, Singer M. Spreading the knowledge on the epidemiology of sepsis. Lancet Infect Dis. 2017;17(11):1104-6.
3. Bozza FA, Salluh Jl. An urban perspective on sepsis in developing countries. Lancet Infect Dis. 2010;10(5):290-1.

4. Machado FR, Cavalcanti AB, Bozza FA, Ferreira EM, Angotti Carrara FS, et al. The epidemiology of sepsis in Brazilian intensive care units (the Sepsis PREvalence Assessment Database, SPREAD): an observational study. Lancet Infect Dis. 2017;17(11):1180-9.

5. Machado FR, Ferreira EM, Sousa JL, Silva C, Schippers P, et al; Latin American Sepsis Institute Network. Quality improvement initiatives in sepsis in an emerging country: does the institution's main source of income influence the results? An analysis of 21,103 patients. Crit Care Med 2017;45(10):1650-1659.

6. Managing ICU surge during the COVID-19 crisis: rapid guidelines. Aziz S, Arabi YM, Alhazzani W, Evans L, Citerio G, Fischkoff K, Salluh J et al. Intensive Care Med. 2020; 8:1-23.

7. Salluh Jl, Lisboa TC. Critical care in Brazil. ICU Manage Pract. 2016;16:3.

8. Brazilian ICUs project.; https://http://www.utisbrasileiras.com.br/en/covid-19/ benchmarking-covid-19/. Accessed 16 June 2020.

9. Writing Group for the CHECKLIST-ICU Investigators and the Brazilian Research in Intensive Care Network (BRICNet), Cavalcanti AB, Bozza FA, Machado FR, Salluh Jl, et al. Effect of a quality improvement intervention with daily round checklists, goal setting, and clinician prompting on mortality of critically ill patients: a randomized clinical trial. JAMA. 2016; 315(14):1480-90.

10. Azevedo LC, Park M, Salluh Jl, Rea-Neto A, Souza-Dantas VC et al.; ERICC (Epidemiology of Respiratory Insufficiency in Critical Care) investigators. Clinical outcomes of patients requiring ventilatory support in Brazilian intensive care units: a multicenter, prospective, cohort study. Crit Care. 2013; 17(2):R63.

11. Soares M, Bozza FA, Angus DC, Japiassú AM, Viana WN, Costa R, et al. Organizational characteristics, outcomes, and resource use in 78 Brazilian intensive care units: the ORCHESTRA study. Intensive Care Med. 2015;41(12): 2149-60.

12. Zampieri FG, Salluh JIF, Azevedo LCP, Kahn JM, Damiani LP, Borges LP, et al. ICU staffing feature phenotypes and their relationship with patients' outcomes: an unsupervised machine learning analysis. Intensive Care Med. 2019:45(11):1599-607.

13. Midega TD, Bozza FA, Machado FR, Guimarães HP, Salluh Jl, Nassar AP Jr, et al. Organizational factors associated with adherence to low tidal volume ventilation: a secondary analysis of the CHECKLIST-ICU database. Ann Intensive Care. 2020;10(1):68

14. Nassar AP Jr, Zampieri FG, Salluh Jl, Bozza FA, Machado FR, Guimarães HP, et al. Organizational factors associated with target sedation on the first $48 \mathrm{~h}$ of mechanical ventilation: an analysis of checklist-ICU database. Crit Care. 2019;23(1):34. https://doi.org/10.1186/s13054-019-2323-y.

\section{Publisher's Note}

Springer Nature remains neutral with regard to jurisdictional claims in published maps and institutional affiliations. 